\title{
Acebutolol and Immediate Release Melatonin in Treatment of Smith-Magenis Syndrome
}

\author{
Benjamin J. Wheeler ${ }^{\mathrm{a}, \mathrm{c}}$, Barry J. Taylor ${ }^{\mathrm{a}}$, Kirsten Simonsen ${ }^{\mathrm{b}}$, David M. Reith ${ }^{\mathrm{a}}$
}

\begin{abstract}
Sleep disturbance occurs in Smith-Magenis syndrome (SMS) as part of the phenotype. It appears that this disturbance lies primarily in an inversion of the circadian rhythm of melatonin. We assessed whether the use of melatonin and acebutolol together would benefit a patient with SMS with regard to sleep and behavior. Our patient entered a four-way crossover, double-blind, double dummy, placebo-controlled n-of-1 trial, using immediate release melatonin, acebutolol and placebo. The duration of the study was 24 consecutive nights. The primary outcome measure was total nocturnal sleep. Placebo/placebo showed the longest total duration of sleep, 10.62 hours (95\% CI: 9.88 - 11.36) as compared to melatonin and acebutolol together with 10.25 (95\% CI: 9.40 - 11.11). There were no significant differences found between the secondary outcome measures in particular behavior or global satisfaction. Our results suggest there is no benefit to sleep or subsequent behavior from adding acebutolol and/or immediate release melatonin in patients with SMS.
\end{abstract}

Keywords: Smith-Magenis syndrome; Melatonin; Acebutolol; Clinical trial

\section{Introduction}

Smith-Magenis syndrome (SMS) is a rare genetic condition characterized by a typical physical phenotype, developmental delay, sleep disturbance and behavioral problems, including self-harm and aggression [1]. The primary defect involves an interstitial deletion on the short arm of chromosome 17 (del 17p11.2). The sleep disturbance of SMS occurs in most pa-

Manuscript accepted for publication March 16, 2015

aDepartment of Women's and Children's Health, University of Otago, Dunedin, New Zealand

bPharmacy Department, Dunedin Public Hospital, Southern District Health Board, Dunedin, New Zealand

'Corresponding Author: Ben Wheeler, Department of Women's and Children's Health, University of Otago, PO Box 913, Dunedin 9054, New Zealand. Email: Ben.wheeler@otago.ac.nz

doi: http://dx.doi.org/10.14740/jmc2102w tients and causes much disruption to the families involved. During the day, this typically involves sleep attacks and daytime napping with disruption of nocturnal sleep [2]. It appears that for many this disruption lies primarily in an inversion of the circadian rhythm of melatonin, a hormone secreted by the pineal gland, causing daytime secretion as opposed to nocturnal [3-5].

Previous case reports have suggested variable benefits with short acting melatonin $[6,7]$. One report showed a reduced time to onset of sleep but little impact on total sleep or subjective measures of behavior [6]. Additionally, a number of case reports have used a slow release melatonin formulation in order to maintain a longer duration of sleep after dosing, in addition to pharmacological suppression of day-time melatonin release with beta blockade, specifically acebutolol $[8,9]$. However, there are not any reports of randomized double-blind placebo-controlled trials aimed at confirming this.

\section{Case Report}

We used a double-blind, n-of-1 study design with randomization via a pre-prepared block randomization schedule over a total duration of 24 consecutive nights. A four-way crossover design was used, with $6 \mathrm{mg}$ of immediate release melatonin, $200 \mathrm{mg}$ acebutolol, and an identical placebo for each, manufactured by our pharmacy in identical capsules. The treatments, in randomized four-night blocks were as follows: acebutolol mane/melatonin nocte (prior to bed), acebutolol/placebo, placebo/melatonin and placebo/placebo. The patient was a 3-year-old boy suffering from SMS confirmed by cytogenetics. His clinical course had previously been complicated by developmental delay, recurrent asthma and obstructive apnoea. Of particular concern to his family was the disrupted pattern of his sleep which exhibited a similar pattern to many of the literature reports (day night sleep reversal and multiple arousals). Because of his recurrent asthma, under supervision in hospital prior to the commencement of the study, a trial of oral acebutolol was conducted to assess any worsening of bronchospasm.

The primary outcome measure was uninterrupted sleep after night-time medication (sleep efficiency). The secondary outcome measures were: total sleep over $24 \mathrm{~h}$, total nocturnal sleep, the number of arousals, and total daytime sleep. Five point ordinal scales were recorded for daytime behavior, pa- 
Table 1. Comparison of Efficacy Outcome Measures Between the Treatments

\begin{tabular}{lllll}
\hline Outcome measure & Acebutolol/melatonin & Acebutolol/placebo & Placebo/melatonin & Placebo/placebo \\
\hline Sleep after pm medication (h), mean (95\% CI) & $8.33(8.06-8.61)$ & $6.49(4.11-8.86)$ & $7.67(5.98-9.35)$ & $7.46(5.65-9.26)$ \\
Nocturnal sleep (h), mean $(95 \%$ CI) & $8.33(8.06-8.61)$ & $7.82(7.26-8.38)$ & $8.37(7.65-9.10)$ & $8.44(7.79-9.10)$ \\
Sleep during day (h), mean $(95 \% \mathrm{CI})$ & $1.92(0.87-2.97)$ & $1.28(1.01-1.54)$ & $1.44(0.85-2.04)$ & $2.18(1.40-2.95)$ \\
Total sleep (h), mean (95\% CI)* & $10.25(9.40-11.11)$ & $9.10(8.69-9.51)$ & $9.82(8.87-10.77)$ & $10.62(9.88-11.36)$ \\
Time to sleep onset (min), mean (95\% CI) & $29.17(14.92-43.41)$ & $45.83(13.59-78.08)$ & $30(17.15-42.85)$ & $27(-9.88-63.88)$ \\
Number of arousals, median (range) & $1(1-1)$ & $1(1-2)$ & $1(1-2)$ & $1(1-2)$ \\
Daytime behavior, median (range) & $1(1-2)$ & $1.5(1-3)$ & $2(1-2)$ & $1.5(1-2)$ \\
Paternal sleep score, median (range) & $3(2-3)$ & $3(3-4)$ & $3(3-3)$ & $3(2-3)$ \\
Maternal sleep score, median (range) & $4(3-5)$ & $4(3-4)$ & $4(3-4)$ & $3.5(3-4)$ \\
Global score, median (range) & $3(3-4)$ & $3(2-4)$ & $3(3-3)$ & $3(3-3)$ \\
\hline
\end{tabular}

${ }^{*}$ P-value $<0.05$. Continuous variables tested using ANOVA. Ordinal variables tested using Kruskal-Wallis.

ternal sleep quality, maternal sleep quality, wheeze and global satisfaction with the treatment. The outcome measures were recorded by the parents in a treatment diary. Overnight pulse oximetry was performed on one occasion for each treatment and measures of sleep time, arousals, sleep stages and oxygenation were derived from these recordings (analyzed blind to treatment status). The data were analyzed using ANOVA and 95\% CIs for continuous, normally distributed variables and the Kruskal-Wallis test for ordinal variables. Full consent for this study was provided by the patient's parents, and full ethical approval was obtained via the Lower South Regional Ethics Committee.

There was no significant difference between the treatments in the amount of sleep after medication (sleep efficiency) (Table 1), although the greatest amount of sleep after medication occurred with the acebutolol/melatonin combination.

A significantly greater amount of total sleep occurred with the placebo/placebo combination than the acebutolol/placebo combination. Although the acebutolol/placebo combination had the shortest duration of daytime sleep, it also had the shortest duration of sleep after medication and total sleep times. In addition, although not statistically significant, the acebutolol/ placebo combination had the greatest mean time to onset of sleep. There was no difference between the treatments in duration of nocturnal sleep, nocturnal arousals, daytime behavior scores, the quality of maternal or paternal sleep, or in global parental satisfaction. Exacerbation of wheeze was not found for any of the treatments.

\section{Discussion}

The present study suggests that for this patient, melatonin and acebutolol in combination have little effect on duration of nocturnal sleep. This is contrary to our expectations which were based on previous reports and our own experience on inducing sleep in SMS. In addition we were not able to demonstrate an improvement in behavior, in the quality of parental sleep or overall satisfaction as a result of treatment. There was no indication of significant respiratory depression with melatonin or exacerbation of bronchospasm with acebutolol suggesting that both treatments were well tolerated.

A previous n-of- 1 study in the same patient suggested a benefit in decreasing sleep latency (the time to onset of sleep) in SMS with short acting melatonin, but no improvement in other outcome measures [6]. A meta-analysis of the effects of exogenous melatonin in adults indicated that melatonin decreases sleep latency, and increases sleep efficiency (the duration of nocturnal sleep) but does not increase the total duration of sleep over a 24-h period [10]. Melatonin is beneficial in treating delayed sleep phase syndrome by decreasing sleep onset latency by a mean of $38.8 \mathrm{~min}$, but has not been demonstrated to be beneficial in insomnia [11]. However there is a possibility that publication bias exists for studies of melatonin in adults [10]. Similarly in children with neurodevelopmental disabilities, melatonin resulted in a decreased time to onset of sleep, but not in an increase in total sleep, a decrease in the number of night time awakenings or increased parental satisfaction [7].

Beta blockers themselves can cause sleep disturbance [12]. These disruptions include nightmares and insomnia. The basis of sleep disturbance in association with beta blockers appears to be disturbance of the secretion of melatonin. The present study suggests, although the finding was not statistically significant, that treatment with acebutolol alone is detrimental, with resulting increased time to sleep onset and reduced durations of sleep. Acebutolol has been shown to suppress the daytime release of melatonin in patients with SMS, and in open, uncontrolled trials has resulted in improvements in sleep efficiency and total duration of sleep $[8,9,13]$. However, measures of sleep duration are subjective and open to interpretation by the parents/carers of the study subjects. Hence the importance of blinding in studies that use sleep diaries to record outcome measures.

The double-blind, randomized controlled design of this n-of-1 study is a considerable strength over previous case reports in SMS, and highlights the potential of this technique in rare disorders. The negative findings of this study are disappointing, and as confined to one patient, generalizability remains an important weakness. In the largest case series to date using this treatment, a variable response was seen, with no 
response in two out of 10 participants, providing support that response can be variable across patients.

In conclusion, despite promising findings from non-blinded case reports for acebutolol and the sleep disruption of SMS, given the lack of demonstrable benefit in this study, and the known adverse effects of beta blockers, questions remain as to its efficacy. Currently, there is insufficient evidence to support the routine use of acebutolol in SMS, other than as part of a larger well-designed study.

\section{Acknowledgement}

We wish to thank the patient and his family for their enthusiastic involvement in this study.

\section{Conflict of Interest}

The authors declare that they have no conflict of interest.

\section{References}

1. Greenberg F, Guzzetta V, Montes de Oca-Luna R, Magenis RE, Smith AC, Richter SF, Kondo I, et al. Molecular analysis of the Smith-Magenis syndrome: a possible contiguous-gene syndrome associated with del(17) (p11.2). Am J Hum Genet. 1991;49(6):1207-1218.

2. Smith AC, Dykens E, Greenberg F. Sleep disturbance in Smith-Magenis syndrome (del 17 p11.2). Am J Med Genet. 1998;81(2):186-191.

3. Cornelissen G, Halberg F, Tarquini R, Perfetto F, Salti R, Laffi G, Otsuka K. Point and interval estimations of circadian melatonin ecphasia in Smith-Magenis syndrome. Biomed Pharmacother. 2003;57(Suppl 1):31s-34s.

4. Novakova M, Nevsimalova S, Prihodova I, Sladek M, Sumova A. Alteration of the circadian clock in children with Smith-Magenis syndrome. J Clin Endocrinol Metab.
2012;97(2):E312-318

5. Kocher L, Brun J, Devillard F, Azabou E, Claustrat B. Phase advance of circadian rhythms in Smith-Magenis syndrome: a case study in an adult man. Neurosci Lett. 2015;585:144-148.

6. Wheeler B, Taylor B, Simonsen K, Reith DM. Melatonin treatment in Smith Magenis syndrome. Sleep. 2005;28(12):1609-1610.

7. Phillips L, Appleton RE. Systematic review of melatonin treatment in children with neurodevelopmental disabilities and sleep impairment. Dev Med Child Neurol. 2004;46(11):771-775.

8. De Leersnyder H, Bresson JL, de Blois MC, Souberbielle JC, Mogenet A, Delhotal-Landes B, Salefranque F, et al. Beta 1-adrenergic antagonists and melatonin reset the clock and restore sleep in a circadian disorder, SmithMagenis syndrome. J Med Genet. 2003;40(1):74-78.

9. Van Thillo A, Devriendt K, Willekens D. [Sleep disturbances in Smith-Magenis syndrome: treatment with melatonin and beta-adrenergic antagonists]. Tijdschr Psychiatr. 2010;52(10):719-723.

10. Brzezinski A, Vangel MG, Wurtman RJ, Norrie G, Zhdanova I, Ben-Shushan A, Ford I. Effects of exogenous melatonin on sleep: a meta-analysis. Sleep Med Rev. 2005;9(1):41-50.

11. Buscemi N, Vandermeer B, Hooton N, Pandya R, Tjosvold L, Hartling L, Baker G, et al. The efficacy and safety of exogenous melatonin for primary sleep disorders. A meta-analysis. J Gen Intern Med. 2005;20(12):11511158.

12. Brismar K, Hylander B, Eliasson K, Rossner S, Wetterberg L. Melatonin secretion related to side-effects of beta-blockers from the central nervous system. Acta Med Scand. 1988;223(6):525-530.

13. De Leersnyder H, de Blois MC, Vekemans M, Sidi D, Villain E, Kindermans C, Munnich A. beta(1)-adrenergic antagonists improve sleep and behavioural disturbances in a circadian disorder, Smith-Magenis syndrome. J Med Genet. 2001;38(9):586-590. 